Original Article

\title{
Comparison of Early vs. Late Tracheostomy in Subdural Hematoma Operated at GCS Six or Below
}

\author{
Rana Zubair Mahmood', Sikandar Ali Dehraj', Sarfraz Khan², Usama Mansoor', Abdul Wajid', \\ Touqeer Ahmad ${ }^{1}$, Waqas Bashir ${ }^{1}$ \\ ${ }^{1}$ Department of Neurosurgery, Punjab Institute of Neurosciences (PINS)/LGH, Lahore - Pakistan \\ ${ }^{2}$ Department of Neurosurgery, Kohat Institute of Medical Sciences, Kohat - Pakistan
}

\begin{abstract}
Objectives: The aim of this study was to compare the outcomes of early tracheostomy vs. late tracheostomy in post-operative patients after acute subdural hematoma at receiving GCS (Glasgow comma scale) of six or below.

Material and Methods: A quasi observational study was conducted on 30 patients with acute subdural hematoma after RTA (road traffic accident) and were operated in The Department of Neurosurgery Unit 2, Punjab Institute of Neurosciences, LGH, Lahore. The age range was $20-65$ years. All patients were operated upon within 12 hours of RTA.
\end{abstract}

Results: In Group A, 12 (40\%) patients, decompressive craniectomy with the evacuation of acute subdural hematoma and early tracheostomy were performed. In Group B, 8 (26\%) patients' craniotomy and evacuation of acute subdural hematoma were done along with early tracheostomy. In 6(20\%) patients, decompressive craniectomy and evacuation were done and their tracheostomies were done at the $10^{\text {th }}$ post-operative day. In $4(13.33 \%)$ patients' craniotomy and evacuation of hematoma done and their tracheostomies were also done at $10^{\text {th }}$ post-operative day. In Group A, on $5^{\text {th }}$ postoperative day GCS of 16 (53.33\%) patients with early tracheostomies and fewer comorbidities improved, they were extubated, while 2 (6.67\%) patients did not improve and 2 (6.67\%) patients died. In Group B, in 30 patients with late tracheostomies, only 4 (13.33\%) patients were improved. On $10^{\text {th }}$ post-op day, GCS of 4 (13.33\%) patients improved, GCS of 3 (10\%) patients not improved and 3 (10\%) patients died.

Conclusion: Early tracheostomy in patients with acute subdural hematoma yields good results as compared to late tracheostomy.

Keywords: Early Tracheostomy, Late Tracheostomy, Acute Subdural Hematoma.

Corresponding Author: Rana Zubair Mahmood

Department of Neurosurgery, Punjab Institute of

Neurosciences (PINS), Lahore - Pakistan

Email: 33ranazubair356@gmail.com
Date of Submission: 10-05-2021

Date of Revision: 26-06-2021

Date of Online Publishing: 30-06-2021

Date of Print: 30-06-2021

DOI: $10.36552 /$ pjns.v25i2.565 


\section{INTRODUCTION}

Placement of tracheostomy tube in a person's trachea by creating an opening in the neck either temporarily or permanently is called tracheostomy. Tracheostomy tube is placed below vocal cords in 3 to 4 tracheal ring and in this way dead space decrease breathing occur by passing mouth, nose and throat. ${ }^{1}$ We have to perform tracheostomy in anaphylaxis, airway burn, neck cancer, chronic lung disorders, diaphragm disorders, infection, trauma to airway, and vocal cord paralysis. $^{2}$

For elective tracheostomy, fasting up to 12 hours is needed while for emergency no need for any preparation. Tracheostomy can be performed in general anesthesia as well as in local anesthesia. For tracheostomy we must know the following landmarks i.e., hyoid bone (C3), thyroid cartilage, cricoid cartilage (C6), thyroid gland, and sternohyoid muscle. For tracheostomy, we have to cut the following layers from outwards to inwards; skin, subcutaneous tissue, fat, pretracheal fascia (superficial and deep), trachea. After tracheostomy, the tube is secured by the band around the neck. Normally, it takes 24 to 72 hours for adoption to breathe through a tracheostomy tube. ${ }^{3,4}$

Tracheostomy can cause damage to the thyroid gland, trachea, can cause lung collapse, and scar tissue. 5,6,7,8 For tracheostomy care, we should inspect the skin around the tracheostomy wound for redness, hardness, tenderness, discharge once daily. Between the tie and your neck, there should be space for two fingers. After making a slit, a fine mesh gauze under the tracheostomy tie. The skin around the stoma must be soaked by cotton-tipped swabs in a solution of half hydrogen peroxide and half water. ${ }^{9}$ Bleeding between the inner layer of the dura mater and the arachnoid mater of the meninges surrounding the brain is called a subdural hematoma. It usually results from tears in bridging veins. ${ }^{10}$ We should evacuate the acute subdural hematoma (SDH) with a thickness greater than $10 \mathrm{~mm}$ or a midline shift greater than $5 \mathrm{~mm}$ on computed tomographic (CT) scan. ${ }^{11}$

A subdural hematoma can cause brain herniation, anxiety memory loss, weakness in the body, numbness, coma, seizures, speaking difficulty, dizziness, headache, and death if complicated. ${ }^{12}$ For subdural hematoma, diuretics, fluid therapy, antiepileptics, and antibiotics are options for medical therapy. ${ }^{12}$ In Decompressive craniectomy a part of the skull is removed is performed on victims of traumatic brain injury, stroke, Chiari Malformation, and other conditions associated with raised intracranial pressure. Daily clinical status and CT scan were the ways to monitor the outcomes. ${ }^{12}$ The current study aimed to compare the outcomes of early tracheostomy vs late tracheostomy in post-operative patients after acute subdural hematoma at receiving GCS (Glasgow comma scale) of six or below.

\section{MATERIAL AND METHODS}

\section{Study Design}

A comparative observational study conducted on 30 patients after a road traffic accident (RTA), and were operated in Neurosurgery Department, Punjab Institute of Neurosciences (PINS) during $1^{\text {st }}$ February 2021 to $31^{\text {st }}$ May 2021. Prior ethical approval was taken from IRB Committee.

\section{Inclusion Criteria}

Male and female patients included who were having acute subdural hematoma with age range $10-70$ years. Those patients included those who were having a GCS of 6 or below, having comorbidities and poly-trauma, having subdural hematoma thickness greater than $10 \mathrm{~mm}$, having midline shift greater than $5 \mathrm{~mm}$ and found in the duration of injury of 12 hours.

\section{Exclusion Criteria}

Patients excluded who had GCS above 6 or had a 
duration of injury of more than 12 hours. Those patients excluded who were in the age less than 20 years, who found with a thickness of subdural hematoma of less than $10 \mathrm{~mm}$ and who had midline thickness less than $5 \mathrm{~mm}$.

\section{Data Collection}

Patients' data were entered on a pre-designed proforma. Informed consent was taken from the patients' attendants for the data. SPSS version 22 was used for the data analysis.

\section{RESULTS}

\section{Age Incidence}

The age range was 20-65 years and mean age was 42.5 years.

\section{Gender Incidence}

There were 25 male patients and 5 were female patients.

\section{Operative Technique}

In all cases, we evacuated subdural hematoma by making a Montreal flap. In our 18 patients, we removed bone for decompressive craniectomy while in 12 patients bone flap placed. In all cases Duraplasty was done. We did tracheostomy by horizontal incision.

\section{Outcome}

Most of the patients (66.67\%) reported a good surgical outcome. Complications and mortality was reported $16.66 \%$. See Table 1.

Table 1: Outcome of Surgery.

\begin{tabular}{lc|} 
Outcome & Number of Patients (\%) \\
Good & $20(66.67 \%)$ \\
Complications & $5(16.66 \%)$ \\
Mortality & $5(16.66 \%)$ \\
No improvement & $5(16.66 \%)$ \\
\hline
\end{tabular}

\section{Post-operative Complications after Decompressive Craniectomy of Subdural Hematoma and Tracheostomy}

In our 3(10\%) patients, subdural hygroma developed which was later managed surgically. In $2(6.67 \%)$ patients, the wound flap got infected which was washed with normal saline and treated with daily dressing and antibiotics according to culture and sensitivity report. In 1 (3.33\%) patient, brain abscess was formed which managed surgically and proper antibiotics.

\section{DISCUSSION}

We included patients of age range $20-65$ years and with a mean age of 42.5 years. In our study, $25(83.33 \%)$ patients were male and 5 (16.67\%) patients were female. The mechanism of injury in 22 (73.34\%) patients were RTA mostly fall from the bike and in 8 (26.66\%) patients were spontaneous after an increase in blood pressure. The patients who had spontaneous acute subdural hematoma were mostly in the age range of $40-65$ years.

Duraplasty was done in all cases and drain was placed postoperatively in all cases in sub-glial position. Co-morbidities in our patients were DM (Diabetes Mellitus), HTN (Hypertension), IHD (Ischemic Heart Disease), and Polytrauma.

The presenting complaints in our patients was the loss of consciousness, multiple episodes of vomiting, ENT bleed, fits, and headache. All patients we operated on within 12 hours of injury or increase of BP. In our set-up usually, patients present late i.e. after 6 hours due to lack of neurosurgical facilities in peripheral hospitals.

In 12 (40\%) patients decompressive craniectomy with the evacuation of acute subdural hematoma and early tracheostomy were performed. In 8 (26\%) patients' craniotomy and evacuation of acute subdural hematoma were done along with early tracheostomy. In 6 (20\%) patients decompressive craniectomy and 
evacuation was done and their tracheostomies were done at $10^{\text {th }}$ post-operative day. In 4 (13.33\%) patients, craniotomy and evacuation of hematoma done and their tracheostomies were also done at $10^{\text {th }}$ post-operative day.

In patients who were presented with spontaneous subdural hematoma, all baseline, ECG, chest X-ray, and bleeding profile were monitored. Their PT (prothrombin time), APTT (activated partial thromboplastin time), INR (international normalized ratio,) BT (bleeding time), CT (clotting time) were monitored and normalized before operation. Whole blood, FFPs (fresh frozen plasma) and megakaryocytes were transfused to normalize the bleeding profile.

In all cases, we performed CT brain plan on presentation to neurosurgical department and repeat scan done after 12 hours of surgery. Post operatively, sedation and paralysis were given to 18 (60\%) patients for 24 hours to normalize ICP (intracranial pressure). Blood pressure monitored were attached and BP was kept in the range of $140-160 \mathrm{mmHg}$.

In our study, we did tracheostomy at the time of primary surgery in $20(66.67 \%)$ patients and late tracheostomy was done in 10 patients on $10^{\text {th }}$ post-operative day. Tracheostomy care was done. We changed the dressing of the tracheostomy and wound care perfectly done. It was seen those 16 (53.33\%) patients with early tracheostomy GCS improved dramatically patients were extubated and they all discharged within 10 days after telling them about tracheostomy care. In 2 (6.66\%) patients GCS not improved and 2 (6.66\%) patients died.

Siempos et al. (2015) mentioned that, fewer complications like pneumonia after early tracheostomy so reduces hospital stay in critically ill patients. $^{13}$ In 2014, Szakmany et al. recommended that there is less duration of sedation after early tracheostomy in critically ill patients. ${ }^{14}$ In 2012, Zheng et al. concluded that early tracheostomy reduces ICU stay and improves outcomes earlier than late tracheostomy in critical ill patients. ${ }^{15}$

Meng et al. mentioned that early tracheostomy reduces hospital duration and does not improve mortality in critically ill patients. ${ }^{16}$ In 2010, Gandía-Martínez et al. did a study and mentioned that an early tracheostomy reduces hospital stay, ICU stays, sedation duration, and antibiotics need in critically ill patients. ${ }^{17}$ Koch et al. reported that early tracheostomy reduces ICU stay, hospitalization but does not improve mortality in critically ill patients. ${ }^{18}$

In patients with late tracheostomy, 4 (13.3\%) patients GCS improved and they discharged within 10 days after telling them tracheostomy care. Three (10\%) patients did not show any improvement and our 3 (10\%) patients died. In our patients, postoperatively surgical site infection occurred in 2 patients and GTCS occurred in 3 patients which were managed later on. The follow-up period was 2 weeks through OPD. Patients were given antibiotics, antiepileptics, pain killers, and anti-emetics. Patients with comorbidities are advised to get them check-up by the medical department also.

\section{CONCLUSION \& RECOMMENDATIONS}

Patients with early tracheostomy showed improvement in clinical status as compared to late tracheostomy. Early tracheostomy of patients with GCS 6 or below results in a better prognosis. There are the following advantages of early tracheostomy: decreased dead space, easy weening from ventilator and lessor hospital stay.

\section{Limitations}

The number of cases was small. Hence large case series for further evaluation by this approach is awaited. 


\section{REFERENCES}

1. Johnson RF. Adult Tracheostomy. Houston, Texas: Department of Otolaryngology-Head and Neck Surgery, Baylor College of Medicine. Archived from the original on 17 May 2008.

2. Lindman JP, Charles E Morgan. Tracheostomy. WebMD. 2010.

3. Lalwani, Anil K. Current Diagnosis \& Treatment in Otolaryngology-Head \& Neck Surgery, 3e. New York, NY: McGraw-Hill. pp. Yu KY. Chapter 38. Airway Management \& Tracheotomy, 2012. ISBN 978-0-07-162439-8.

4. Ellison, E. Christopher; Zollinger, Jr, Robert M. Zollinger's Atlas of Surgical Operations, 10th edition. New York, NY: McGraw-Hill. pp. Chapter 120 - Tracheotomy, Chapter 121 - Tracheotomy, Percutaneous Dilational. ISBN 978-0-07-179755-9. 2016.

5. Simon $M$, Metschke $M$, Braune SA, Püschel K, Kluge $S$. Death after percutaneous dilatational tracheostomy: a systematic review and analysis of risk factors". Critical Care, 2013; 17 (5): R258.

6. Klemm E, Nowak AK. Tracheotomy-Related Deaths. Deutsches Ärzteblatt International, 2017; 114 (16): 273-279.

7. Grant CA, Dempsey G, Harrison J, Jones T. Tracheo-innominate artery fistula after percutaneous tracheostomy: three case reports and a clinical review. British Journal of Anaesthesia, 2006; 96 (1): 127-31.

8. Rajendram R, Khan MF, Joseph A. Tracheostomy tube displacement: an update on emergency airway management. Indian Journal of Respiratory Care, 2017; 6 (2): 800.

9. Subdural hematoma: Medline Plus Medical Encyclopedia. Available: NIm.nih.gov. 2012-06-28.

10. Sanders MJ and McKenna K. Mosby's Paramedic Textbook, 2nd revised Ed. Chapter 22, Head and facial trauma. Mosby. 2001.

11. Bullock MR, Chesnut R, Ghajar J, Gordon D, Hartl R, Newell DW, Servadei F, Walters BC, Wilberger J. Surgical management of traumatic brain injury. Neurosurgery, 2006; 58 (3): 16-24.

12. Papa L, Goldberg SA. Head trauma. In: Walls RM,
Hockberger RS, Gausche-Hill M, eds. Rosen's Emergency Medicine: Concepts and Clinical Practice. 9th ed. Philadelphia, PA: Elsevier. 2018.

13. Siempos II, Ntaidou TK, Filippidis FT, Choi AM. Effect of early versus late or no tracheostomy on mortality and pneumonia of critically ill patients receiving mechanical ventilation: a systematic review and meta-analysis. The Lancet Respiratory Medicine, 2015 Feb. 1; 3 (2): 150-8.

14. Szakmany $T$, Russell P, Wilkes AR, Hall JE. Effect of early tracheostomy on resource utilization and clinical outcomes in critically ill patients: metaanalysis of randomized controlled trials. British journal of anaesthesia, 2015 Mar 1; 114 (3): 396405.

15. Deng H, Fang Q, Chen K, Zhang X. Early versus late tracheotomy in ICU patients: A meta-analysis of randomized controlled trials. Medicine, 2021; 100 (3).

16. Meng L, Wang C, Li J, Zhang J. Early vs late tracheostomy in critically ill patients: a systematic review and meta-analysis. The Clinical Respiratory Journal, 2016; 10 (6): 684-92.

17. Gandia-Martinez F, Martínez-Gil I, Andaluz-Ojeda D, Bobillo de Lamo F, Parra-Morais L, DíezGutiérrez F. Analysis of early tracheostomy and its impact on development of pneumonia, use of resources and mortality in neurocritically ill patients. Neurocirugia (Asturias, Spain), 2010; 21 (3): 211-21.

18. Koch $T$, Hecker B, Hecker A, Brenck F, Preuß $M$, Schmelzer T, Padberg W, Weigand MA, Klasen J. Early tracheostomy decreases ventilation time but has no impact on mortality of intensive care patients: a randomized study. Langenbeck's Archives of Surgery, 2012; 397 (6): 1001-8.

19. Jiang, J.-Y., Xu, W., Li, W.-P., et al. Efficacy of Standard Trauma Craniectomy for Refractory Intracranial Hypertension with Severe Traumatic Brain Injury: A Multicenter, Prospective, Randomized Controlled Study. Journal of Neurotrauma, 2005; 22: 623-628. https://doi.org/10.1089/neu.2005.22.623 


\section{Additional Information}

Disclosures: Authors report no conflict of interest.

Ethical Review Board Approval: The study was conformed to the ethical review board requirements.

Human Subjects: Consent was obtained by all patients/participants in this study.

Conflicts of Interest:

In compliance with the ICMJE uniform disclosure form, all authors declare the following:

Financial Relationships: All authors have declared that they have no financial relationships at present or within the previous three years with any organizations that might have an interest in the submitted work.

Other Relationships: All authors have declared that there are no other relationships or activities that could appear to have influenced the submitted work.

\section{AUTHORS CONTRIBUTIONS}

\begin{tabular}{|l|l|l|}
\hline Sr.\# & Author's Full Name & Intellectual Contribution to Paper in Terms of: \\
\hline 1. & Usama Mansoor & Study design and methodology. \\
\hline 2. & Rana Zubair Mahmood & Paper writing, referencing. \\
\hline 3. & Sarfraz Khan & Literature review, reading. \\
\hline 4. & Abdual Wajid & Data collection and calculations. \\
\hline 5. & Sikandar Ali Dehraj & Analysis of data and interpretation of results etc. \\
\hline 6. & Touqeer Ahmad & Literature review and manuscript writing. \\
\hline 7. & Waqas Basir & Analysis of data and quality insurer. \\
\hline
\end{tabular}

